\title{
The Teaching of Chinese as a second or foreign language:
}

A systematic review of the literature 2005-2015

This paper reports the results of a review of research articles on the teaching of Chinese as a second or foreign language published in four leading mainland Chinese journals during the years 2005-2015. The review found that Chinese language researchers are exploring a wide array of issues including language policy and planning, language learning and use, language pedagogy, teacher development and language testing. These studies report the efforts that Chinese language researchers and teachers have made to meet the explosively growing demand for Chinese language learning in the world. Furthermore, it was noted that the leading Chinese journals have become more receptive to empirical studies although a large number of non-empirical articles are still being published. Overall, research in these journals is still beset with different challenges, and there is an urgent need for more rigorous scholarship on the part of researchers. For this reason, the review concludes with suggestions for Chinese journals so that they can promote high-quality research to support the development of Chinese language education.

Keyword: Chinese language education, non-Chinese learners, multilingualism, language internationalization, language pedagogy 


\section{Introduction}

Foreign language teaching and learning in most contexts means the teaching and learning of English because of its de facto global language status. Likewise, research on language education has long been dominated by studies on the teaching and learning of the English language published in English, which reflects the status of English as a global language and helps perpetuate its powerful dominance in the world. Such domination inevitably does not reflect the multilingual and multicultural realities of the world. For this reason, efforts have been made to diversify the coverage of languages in language education research. For instance, some leading applied linguistics journals (e.g. The Modern Language Journal) stress that they welcome submissions on languages other than English or publish multilingual abstracts to help non-English readers (e.g. Language and Intercultural Communication). Major journal indexes such as the Arts and Humanities Citation Index include journals that publish works in languages other than English. Unfortunately, Asian languages such as Chinese and Japanese are still noticeably underrepresented in these major journal indexes even though these languages, in particular Chinese, have been attracting increasing attention.

To facilitate interaction with other countries and promote participation in globalization since its opening up in the late 1970s, China has made tireless efforts to improve indigenous individuals’ English competence (Gao, Liao, and Li 2014; Hu 2005; Wang and Gao 2008). In the last decade, however, the domination of the English language has been increasingly challenged by the growing importance of Chinese, which is increasingly being taught as an important second or foreign language in and outside China (Moloney and Xu 2015). In China, the government has been expanding its international programmes and is planning to 'attract 500,000 foreign students by 2020, almost three times the ' 140,000 foreign students in 2005' (Zhao, February 27, 2015). In 2004, the first Confucius Institute was launched in

South Korea, and in 2005, the first World Chinese Congress was held in Beijing (中

国网, September 3, 2009). By December 2015, 1,500 Confucius Institutes and classrooms had been launched in 135 countries to teach Chinese to over 1.39 million students (Hanban 2016). Such a dramatic expansion of Chinese as a second or foreign language (CSL/CFL) education has been facilitated by intensified government investment, and it has also been supported by growing research activities covering 
critical issues related to the teaching of Chinese as a second or foreign language (TCSL/TCFL). Much of this research has been conducted by Chinese scholars and published in Chinese journals. Among language learning and teaching researchers, it has often been assumed that researchers in the field of TCSL/TCFL could learn much from researchers in the teaching of English as an international language since English is a predecessor to Chinese in achieving global eminence. However, such an assumption is highly problematic since little is known about what Chinese researchers have been doing to address critical issues in TCSL/TCFL. In fact, what critical issues researchers are concerned with and what they have achieved in the field of CSL/CFL teaching are not adequately known. For these reasons, a review of studies on CSL/CFL teaching and learning published in Chinese journals is pivotal since these studies are published in Chinese and therefore not accessible international readers with no knowledge of Chinese. In the light of similar reviews of foreign language learning and teaching research in China and beyond, this review aims to identify the methodological trends and topical concerns in Chinese journals that publish research on the teaching and learning of Chinese as a second or foreign language from 2005 to 2015. This review attempts to answer the following questions:

1) What methodological trends can be identified in studies on the teaching and learning of Chinese as a second or foreign language (CSL/CFL)?

2) What topical concerns can be identified in studies on the teaching and learning of Chinese as a second or foreign language (CSL/CFL)?

Before we report on the review, we believe it is necessary to outline the widely recognized challenges in the field of TCSL/TCFL. Chinese is a tone language acoustically, and standard Chinese Mandarin has four tones. Chinese is also regarded as a logographic writing system because its written symbols (characters) represent lexical morphemes rather than individual phonemes (Perfetti and Dunlap 2008). Hanyun Pinyin is used to spell syllables for Chinese characters, and complete syllables comprise initials, finals and tone marks. With respect to Chinese characters, which can be divided into integral characters and compound characters based on their physical structure, stokes serve as basic components. About $90 \%$ of Chinese characters are semantic-phonetic compounds, in which one radical signifies the meaning of the compound and the other suggests the sound of the compound (Zhang 1992). In addition, linguistically, Chinese characters, words and vocabulary signify 
different concepts separately. According to Shen and Jiang (2013), as Chinese lacks sound-to-grapheme correspondence, character identification, word segmentation and lexical access are the three fundamental aspects influencing the learning of Chinese, especially reading and writing. Generally speaking, CSL/CFL learners are expected to master the sounds, shapes and meanings of 3,000 high-frequency characters, while they also need to acquire automatic character recognition and production skills in reading and writing. Furthermore, the syntactic relations in modern written and spoken Chinese are represented by means of word order and functional words, which is also a significant challenge for learners. Therefore, Chinese language teachers and researchers have been making strenuous efforts to explore how to facilitate CSL/CFL learners' development of Chinese characters, vocabulary and grammar as well as their appropriate and effective use of Chinese.

\section{The Review}

Given the socio-cultural and historical differences in TCSL/TCFL and the number of Chinese journals in the world, as well as limitations of space in this article, the review was confined to journals published in mainland China, excluding Taiwan, Hong Kong and Macau. Moreover, because of our concern for the potential impact of the relevant studies in the field, we restricted our review to journals listed in the China Social Sciences Citation Index (CSSCI): core journals where CSL/CFL teachers, researchers and policy makers in China compete to publish their research. In total, four leading journals related to TCSL/TCFL were identified, and they all report high impact factors in the CSSCI system (see Table 1).

\section{Insert 'Table 1 Journals reviewed' here}

We decided to focus on publications during the years 2005 to 2015 because the year 2005 witnessed the First World Chinese Congress, a significant event marking China's ambition to promote the teaching and learning of Chinese globally. In addition, we chose papers on TCSL/TCFL for review. A total of 909 articles were identified from the four journals.

To address the first research question, we first read the abstracts of the 909 papers and examined the relevant methodological sections or descriptions in each article. We then decided whether an article was based on empirical research or whether it should be classified as a non-empirical paper. Last but not least, we analyzed the relevant 
methodological descriptions of articles based on empirical research to determine which methodological approach was adopted. To address the second research question, we analyzed the titles and abstracts of the 909 papers to ascertain the topics that each of these papers addressed. Whenever necessary, we also consulted entire papers to determine their topics. On the rare occasions when we were unable to reach a consensus on which topic a paper should be classified under, we followed the classification chosen by the majority of the team. Through this classification process, we identified the following categories: language policy and planning, developments in Chinese language curricula and teaching pedagogy and the impact of these developments on the learning of CSL/CFL, the professional development of Chinese language educators, and the development of Chinese language proficiency tests.

\section{Methodological trends}

Through analyzing article abstracts and relevant methodological information, we identified a total of 424 non-empirical studies and 485 empirical studies in the four journals during the period 2005-2015 (see Table 2). As can be seen in the table, Chinese journals too have been increasingly publishing empirical, particularly quantitative, studies over the years during the review period, such as journals on foreign language education (Gao, Liao and $\mathrm{Li}$ 2014). A closer look at the methodological approaches adopted in the 485 empirical studies revealed that the majority of empirical studies involved quantitative research with only a small number of qualitative and mixed method studies. Most of the empirical studies typically reported the use of statistical analysis or measured language phenomena through methods that included corpus analysis. This finding suggests that relevant research on TCSL/TCFL has been dominated by a positivist paradigm that views language teaching and learning as an objective 'reality' to be known and explained scientifically (Gao, Li, and Lü 2001). It also means that many researchers hope to generalize their findings 'to a much broader population' in an effort to explore issues and phenomena in Chinese language teaching and learning (Lochmiller and Lester 2016, 12).

Insert ‘Table 2 Methodological trends (2005-2015)' here

Although the leading journals on TCSL/TCFL seem to have become much more receptive to empirical studies, a large number of articles in these leading Chinese 
journals are still non-empirical (see Table 3, 46.6\% in total). Although it is possible that some of these articles were based on empirical research, they were excluded from our consideration because the research methodology was poorly described (e.g. Mao 2010; Yang 2010; Zhang, Yang and Zhang 2008). A few articles (17 out of 909) read like general introductions rather than empirical studies because they do not include enough information on data collection and analysis. In addition, the leading Chinese journals on Chinese language education still publish papers that seem to be personal experiences and reflections '[without] substantial literature review, purposeful research planning, details of operational procedure and solid data' (Gao, Li, and Lü 2001, 3). We speculate that these observed phenomena may have to do with relevant researchers' research background and expertise.

\section{Insert 'Table 3 Methodological trends in four journals' here}

We found from the analysis that quite a few researchers (32 out of 909), who claimed their studies constituted experimental research, included no treatment group or control group. Some of them might have confused experimental research designs with causal-comparative designs (e.g. Zha and Wu 2014; Zong, Zhu, and Liu 2012). We also noted that a number of empirical studies were questionable in terms of methodological rigour (29 out of 909). For example, in the 'mixed-method' studies, we were puzzled by the fact that some researchers had reported data collected through only one method (e.g. Ding 2007; Wu and Chen 2012). This observed phenomenon might have to do with constraints such as limitations of space and requirements from powerful gatekeepers including editors and reviewers. However, it is also possible that the relevant researchers may need to improve their research expertise (e.g. research methodology) so that they can conduct rigorous research to inform the teaching and learning of Chinese as a second or foreign language.

\section{Thematic Distributions in the studies}

The thematic analysis revealed that the 909 studies could be categorized into five groups: language pedagogy (398 studies), language learning and use (357 studies), language policy and planning (54 studies), language testing (52 studies) and teacher development (48 studies) (See Appendix 1). The analysis confirmed to some extent the assumption that research on TCSL/TCFL has drawn on relevant research on the teaching and learning of English as an international language. We noticed that many 
studies used concepts and theories that have been explored in research on English language learning and teaching. For example, Tian (2012) analyzed the Master of Teaching English to Speakers of Other Languages (MTESOL) curricula in 12 universities in the UK and contended that these MTESOL curricula could inform the development of Master of Teaching Chinese to Speakers of Other Languages (MTCSOL) programmes. Researchers have also made efforts to test and adapt well-established theories in research on the learning and teaching of English and other languages for use in the context of the teaching and learning of Chinese as an international language. For instance, Su (2010) and Su and Lu (2010) explored the appropriateness of construction grammar theory in advancing a construction-chunk approach to teach grammatical constructions to non-Chinese learners.

In addition, most of the reviewed studies concern the teaching and learning of Chinese in tertiary institutions, and very little research has been conducted at the primary or secondary level. This indicates that TCSL/TCFL remains a major concern for Chinese language teachers in Chinese universities. However, more and more learners are starting to learn Chinese at a young age all over the world. By the end of 2015, over 1,000 Confucius classrooms had been established in primary and secondary schools outside China (Hanban 2016). It is crucial that Chinese language educators undertake inquiries into CSL/CFL learners at various educational levels. For this reason, we also highlight relevant studies on the teaching and learning of Chinese in primary schools and other underrepresented settings in this review.

In light of such general observations, we now discuss relevant studies under the five categories, first focusing on teachers' classroom instructional practices and professional development, and then discussing studies on Chinese language learners' experiences and processes of learning and using the language. Finally, we discuss related studies on language policy and planning and Chinese language proficiency tests.

\section{Language pedagogy}

We were particularly impressed by the large number of studies (398 out of 909) in which Chinese language teachers incorporated new concepts, theories and pedagogical approaches to help CSL/CFL learners acquire proficiency in the language (e.g. Shao 2013; Zong, Zhu, and Liu 2012; Zu 2008). The noticeable presence of research on language pedagogy might have to do with the fact that the rising number of Chinese language learners presents significant pedagogical challenges for 
researchers and teachers. These studies have documented the efforts of Chinese language teachers to develop tailor-made instructional programmes - such as Chinese language courses for specific purposes - for learners who come to China with diverse motivations and needs (e.g. Wang 2005; Ni 2007; Mao 2010; Shen 2006, 2014; Tao 2012; Zhang 2006; Zhao 2008). Given the important role of teaching materials in language education, 95 studies examine how textbooks, dictionaries and other teaching resources can be appropriately designed and effectively used to promote CSL/CFL teaching and learning (e.g. Hao 2013; Li and Gong 2015; Zhou and Chen 2013).

\section{Curriculum development (13 studies)}

Researchers were aware of the necessity to conduct needs analysis before developing any tailor-made programmes. As an example, Ni (2007) surveyed 669 students and 110 teachers to analyze the needs of international students learning Chinese in China. The students were found to prefer to learn Chinese with classmates from different countries having similar Chinese proficiency, and group cooperative learning was their preferred learning style. Ni (2007) also discovered that the students expected Chinese language teachers to provide them with instant feedback in the classroom and wanted to improve their spoken Chinese through practice in real-life situations outside the classroom. Informed by similar needs analysis, Wang (2005) argued that Chinese language curricula should be more flexible and creative in terms of pedagogical content, pedagogical approaches, assessment perspectives and textbook design and use these according to language learners' needs. Liu (2009) proposed that a new Chinese listening curriculum should be designed with the aim of optimizing learners' language communicative competence. In light of English for Special Purposes (ESP) theory, Dong and Han (2014) explored how to construct a specialized Chinese curriculum that prepares overseas students for academic studies in China, which in theory should have the following components: Chinese language element analysis, language function analysis, contextual analysis in specialized areas, and the relationship between Chinese for special purposes and Chinese for general purposes.

\section{Classroom teaching practices (290 studies)}

Classroom teaching practices are critical to the successful implementation of the relevant curricula. A large number of studies (290 out of 398) explored how various pedagogical approaches and practices helped CSL/CFL learners to 'overcome "the 
most difficult language learning” hurdle’ (Yu 2012, 38). For instance, Chen, Ye and $\mathrm{Wu}$ (2015), Jiang (2007) and Xu and Yao (2014) deserve special mention because they examined a highly controversial but under-examined issue related to the integration of Chinese character teaching into Chinese language education. Focusing on learners in primary schools, Chen, Ye and Wu (2015) investigated the impact of Chinese language teaching approaches on learners' orthographic awareness and found no significant difference between the learners in the language-character integration group and those in the language-character separation group. Jiang (2007) explored the effects of separating Chinese character recognition from writing in teaching and of Chinese character recognition-writing integration on CSL/CFL beginners. The Chinese character recognition test results showed that the students in the recognition-writing separation group performed much better in recognizing and writing Chinese characters. Xu and Yao (2014) discussed the relative influence of the language-character integration teaching approach and the language-character separation approach. After one semester of teaching, the results indicated that learners in the language-character separation group had significantly stronger integrative motivation than learners in the other group.

Other studies have also explored the effectiveness of different approaches to teaching pedagogical content. Shao (2013) discussed the effectiveness of the cognitive-functional approach in teaching Chinese personal anaphora, and the results suggested that there was significant immediate and delayed impact on students' acquisition of personal anaphora. Zong, Zhu and Liu (2012) contended that the 'Length approach', in which the length of writing gradually increases in successive tasks for students, was an effective means of helping foreign students to write better compositions. Although the researchers claimed that they had used the new pedagogical approach for six years with ten teachers and also used multiple data-collection instruments to verify the approach, we did not see the research design or supportive evidence at all; hence, this paper was classified as a non-empirical study. At the same time, this research was carried out within one institution setting, and it is not certain whether the relevant findings can be applied to other Chinese language learners in other contexts.

\section{Textbooks, dictionaries and other teaching materials (95 studies)}

Considerable efforts have been made to examine language textbooks as a means of implementing relevant language curricula and guiding pedagogical practices. For 
instance, Hao (2013) explored the authenticity, typicality and appropriateness of elementary Chinese language textbooks, and their findings showed that there was still a pressing need to improve the quality of Chinese language textbooks. While most of the relevant studies examined textbooks for university students, Cai's (2011) study was one of the few that investigated the Chinese textbooks used by primary school students. The study found that more than ten textbooks were used in 163 schools, and the majority of them were designed without specific target learners and even of low quality. Zhou, Luo and Zhang (2010) analyzed nine Chinese culture textbooks used in mainland China or overseas and found that some of these textbooks did not have a clear understanding of the target learners and thus could not provide appropriate cultural content accordingly.

Besides textbooks, some studies have focused on other teaching materials. Through questionnaires and interviews, Hao and Wang (2013) investigated American students' requirements and their teachers' views of Chinese language learners' dictionaries. They found that the learners preferred to use an English-Chinese dictionary and needed a high-quality learner-oriented dictionary. Moreover, the learners needed to be trained to use a dictionary effectively. Peng (2012) also discussed the importance of Chinese newspapers in Chinese language teaching in Malaysia, which could play a special role in promoting Chinese culture and Chinese learning for heritage Chinese language learners.

\section{Language learning and use}

Apart from efforts to develop appropriate curricula, pedagogical practices and materials, researchers have been very concerned with how Chinese language learners learn and use the language. The learning of Chinese has been often regarded as a challenging task for students from both Western countries and Confucian heritage countries (e.g. Japan, Korea and Vietnam) since 'Chinese is fundamentally different from alphabetic languages in terms of phonology, orthography and morphology' (Shen and $\mathrm{Xu}$ 2015, 82). In the review process, we noted that researchers have investigated CSL/CFL learners' learning process and features of their Chinese character, phonetic, lexical and grammatical development. They have also paid great attention to improving these learners' ability to use both spoken and written Chinese effectively, reflecting the impact of communicative language teaching approaches on CSL/CFL teaching, especially in the last five years. Some studies have also examined factors that differentiate individual learners' learning and use of Chinese, including 
attitudes, motivations, beliefs and strategy use in learning and using Chinese. Before discussing these themes in detail, we offer the following observations concerning this group of studies:

1. Most of the studies portray non-Chinese learners' learning as a linear and unidirectional process towards native-like performance. For this reason, learners' first languages are commonly considered as a source of negative impact on their efforts to 'master' the Chinese language (e.g. Feng and Hu 2005; Huang et al. 2005; Yuan 2009).

2. Researchers have greatly favored particular methodological approaches, such as corpus analysis (e.g. Liu 2006; Xiao and Liu 2013; Xie 2010; Zheng 2015; Zhu 2007), when exploring the learning processes and developmental features of CSL/CFL learners. As mentioned earlier, although researchers have attempted different research approaches, some of them have had a very vague understanding of experimental research designs and have often confused them with causal-comparative or correlational designs. The number of studies with such a problem (17 out of 909) suggests that some researchers might need to improve their methodological understanding in undertaking research.

CSL/CFL learners' Chinese character (24 studies), phonetic (44 studies), lexical and grammatical development (166 studies)

Learning Chinese characters presents an enormous challenge for many CSL/CFL learners. Wu et al. (2006) found that European and American students were worse at recognizing and writing Chinese characters than Koreans and Japanese. Korean, European and American students made more errors in writing near homograph characters, but Japanese students made more errors in writing near homophone characters. This indicates that different CSL/CFL learners face their own unique challenges in learning Chinese characters despite some of them having a first language closer to or more distant from the Chinese language.

Researchers have also displayed a strong interest in Chinese language learners' phonetic acquisition. Wen (2010) confirmed that American students' learning of seven basic vowels follows the order of $\mathrm{i}>\mathrm{y}, \mathrm{\imath}, \mathrm{\imath}>\mathrm{u}$, a, ə, contradicting Lado's (1957, cited in Wen 2010) contrasting analysis hypothesis that assumes learners' linguistic development to be linear or unidirectional. Mei (2011) observed the Chinese affricate learning process of Thai speaking learners and reported the strong effect of first language transfer on Chinese acquisition. Using a variety of data collection methods 
such as questionnaires, interviews and classroom observations, An and Zhang (2007) discovered that students from countries using Chinese characters identified Pinyin faster than did those from countries using no Chinese characters. Chen (2011) examined the tone acquisition process of Western learners at different levels and found the learners' tone performances changed with their Chinese language proficiency levels.

With regard to lexical development (37 out of 166), Zhang (2008) examined the effect of stroke number, word frequency and morpheme frequency on Chinese one-character word recognition. The results suggested that it was harder for learners to memorize Chinese characters with more strokes, confirming that the stroke is a primary unit in character recognition. Fan (2013) noted that CSL/CFL learners are inclined to learn neologisms with more practicality, timeliness and productivity and to acquire new words through daily communication with Chinese, TV programmes, films and the internet. Ding (2006) examined the learning order of three Chinese interrogative pronouns Shenme, Nar and Shui through corpus analysis, and also found that language use frequency was consistent with the learning order. Such research helps language educators construct relationships between word forms and word meanings when teaching Chinese vocabulary.

Since Chinese syntactic relations are often represented by means of word order and functional words, unlike those in many other languages (Huang and Liao 2002), CSL/CFL learners' syntactic development has received substantial attention in research (129 out of 166). Researchers have extensively explored CSL/CFL learners' acquisition of challenging constructions such as those involving $\mathrm{Ba}, \mathrm{Bei}$ and $\mathrm{Bi}$ through corpus analysis (e.g. Huang and Xiao 2012; Li and Deng 2005; Mo 2007; Peng 2008; Wang 2005). Huang et al. (2007) found that English-speaking learners tended to use Bei to display completion states, and that their first language affected Chinese passive patterns learning. Huang and Xiao (2012) contended that the acquisition order of the $\mathrm{Ba}$ construction could be a practical guideline for teaching students at different stages. CSL/CFL learners' learning of other complex grammatical structures such as prepositional phrases has also been investigated in studies such as that of Lin (2011), who noted that Koreans learn different sentences with Zai +Noun +Location in a particular sequence. Luan (2013) identified that the majority of students in the HSK dynamic composition corpus could not distinguish Gei as a verb from Gei as a preposition word. Therefore, Luan suggested that educators teach the Gei construction using chunking theory. 


\section{Features of learner Chinese (81 studies)}

A substantial number of studies have documented the linguistic features of CSL/CFL learners' written and spoken Chinese. For instance, Dai (2007) found that learners used more self-repair than other-repair in spoken Chinese and tended to use other-initiated self-repair in the self-repair in authentic daily dialogues. $\mathrm{Li}$ and $\mathrm{Xu}$ (2009) found that learners often misused the negative adverbs $\mathrm{Bu}$ and $\mathrm{Mei}$ when talking about past actions and events in Chinese. Chen (2015) found that American students' oral Chinese development was unbalanced in terms of complexity, accuracy and fluency, and that internal competition and cooperation coexisted between the three dimensions, creating challenges for the students' language development. An (2015) concluded from his analysis of the HSK dynamic composition corpus that high-achieving learners performed better in word fluency and syntactic complexity but not in accuracy. In contrast, Yuan (2009) noticed that errors of omission and logical relations were common among English-speaking Chinese language learners in her corpus. She therefore argued that it would be more effective for educators to teach cohesive Chinese words in a discourse context

\section{Leaning attitudes, motivation, awareness and strategies (42 studies)}

Researchers have explored how individual difference factors such as attitudes, motivation, beliefs and strategies mediate CSL/CFL learners' learning and use of the language. With regard to CSL/CFL learners' motivation to learn the language, Zhang (2015) identified a significant correlation between American students' motivation and their Chinese language learning. Ding (2014) noticed that CSL/CFL learners' motivation was not particularly strong, and that motivated learners largely referred to individual interest and experiences of Chinese culture as key factors that underpinned their learning efforts. This suggests that engaging learners with motivating experiences with Chinese culture can help foster more interest in learning the language.

Given the uniqueness and complexity of Chinese language acquisition, researchers have also explored strategies employed by CSL/CFL learners and the factors underpinning their strategy use. Liu (2012) found that Japanese university students learning Chinese used a memory strategy the most and emotional and functional strategies (e.g. watching Chinese movies and interacting with Chinese friends) the least. At the same time, students' learning motivation and learning 
strategy use had a positive correlation. Wu and Chen (2006) found that students with better listening competence adopted better cognitive, metacognitive and emotional strategies in listening to Chinese and suggested that CSL/CFL teachers should encourage students to take the initiative and provide more self-assessment tasks when teaching listening. Zhou and Xie (2007) explored the word separation strategies that intermediate level students applied through reading tasks and stimulated recall since Chinese word segmentation is a complicated task for the majority of CSL/CFL learners. They concluded that using familiar words, grammatical judgment, rhythmical judgment, substitution and native language understanding were common strategies in Chinese lexical extraction. Qian (2010) found that Korean students understood Chinese readings mostly through main idea speculation, context information and text marking but avoided using first language and interactive strategies. In contrast, Wu (2008) discovered that Italian students mostly adopted compensation and communication strategies to improve their oral Chinese in the target-language environment.

\section{Language policy and planning, language testing and teacher development}

This group of 154 articles addressed the critical issues of language policy and planning, language testing and teacher development at the heart of the global promotion of Chinese language learning and teaching. As mentioned earlier, 54 of them examined the expansion of TCSL/TCFL at a macro policy and planning level. Fifty-two of them explored appropriate and reliable ways of assessing Chinese language learners' learning achievement, while 48 studies discussed how Chinese language teachers can be prepared for the task of teaching CSL/CFL more effectively.

\section{Language policy and planning (54 studies)}

The attention that CSL/CFL teaching and learning has received in the reviewed studies has been instigated by changes at the policy level. As reflected in the studies on relevant language policy and planning, policymakers and academic researchers share the same goal as they

'devote themselves to satisfying the demands of people from different countries and regions in the world who learn the Chinese language, to enhancing understanding of the Chinese language and culture by these people, to strengthening educational and cultural exchange and cooperation between China and other countries, to deepening friendly relationships with other nations, to 
promoting the development of multiculturalism, and to constructing a harmonious world.' (Constitution and By-laws of the Confucius Institutes: General Principles)

These articles report on the teaching and learning of Chinese in Asian countries such as Bangladesh (Gong, Ding, and Chen 2008), Indonesia (Cai 2009), Korea (Lei 2006; Yang 2005), Japan (Guo 2005), Singapore (Chin 2013) and Thailand (Chen 2006; Huang 2005; Li 2010; Wang 2008; Wu and Yang 2008). They also document efforts to promote the teaching and learning of Chinese in Western countries including Australia (Chen 2013), Brazil (Chen 2015), Canada (Cui 2005; Li 2005), Columbia (Zhang 2008), France (Bellassen and Liao 2013), Germany (Geng 2005), and the USA (Liu 2014; Qian 2011; Wang and Chu 2009; Wen 2011; Yao 2014; Zhao, Zhang, and Yao 2013). These reports show that Northeast Asia (including Japan and Korea) has become one of the most active regions in CSL/CFL teaching and learning because of socio-cultural exchanges and its geographical proximity to China (Cao 2008). Chen (2013) contended that Australia's language policy and overseas Chinese heritage language students were two important factors underpinning the teaching of CSL/CFL in the country. Wu and Yang $(2008,128)$ highlighted the dramatic growth in the number of Chinese language learners (a quarter of million by the end of 2006) in Thailand, which was 'a miracle in the international spread of Chinese.' As was mentioned earlier (also see Appendix 1), the majority of these articles (52 of 54) reported non-empirical studies. In these articles, the researchers merely surveyed the situation concerning TCSL/TCFL in different countries and speculated what factors might have mediated the promotion of Chinese teaching and learning. Nevertheless, policymakers and curriculum developers may find these reports useful when developing Chinese language curricula for specific countries.

\section{Language testing (52 studies)}

Given the rapid expansion of TCSL/TCFL worldwide and the fact that Taiwan is a competitive provider of Chinese language education (Dai and Yang 2012), there is an urgent need for the authorities concerned in mainland China to construct, develop and promote widely approved standards to evaluate teaching and learning Chinese as an international language (CIL). Wang $(2008,71)$ observed that 'it should become very hard and challenging if there is no internationally approved standard in learning and teaching Chinese as an international language' after examining foreign language education criteria in the USA and EU. For this reason, policymakers and researchers 
propose to contextualize the foreign language education standards from other nations (such as the Standards for Foreign Language Learning in the 21st Century [2006] and the Common European Framework of Reference for Languages: learning, teaching, assessment [2001]) in TCSL/TCFL. In addition, researchers have also proposed an array of practical directions for the teaching and learning of CIL. For instance, Li (2015) insisted that Hanyu Pinyin, Putonghua and simplified Chinese characters should all be the components of standardized CSL/CFL curricula in China as well as abroad.

Substantial efforts have been put into developing appropriate and reliable standard Chinese language examinations, such as the more appropriate and effective Hanyu Shuiping Kaoshi (HSK), the Business Chinese Test (BCT) and the Spoken Chinese Test (SCT). According to Hanban (2016), six million learners took these Chinese language examinations around the world in 2015. As a result, researchers are quite concerned with the validity, reliability and operationalization of scoring scales in these standardized language tests. Therefore, Wang (2006) examined the external validity of the HSK and concluded that it has high validity with regard to its evaluation of learners' Chinese language performance. Li and Li (2014) reached similar conclusions with regard to the SCT’s validity and reliability.

\section{Teacher development (48 studies)}

Since language teachers play a critical role in implementing relevant language curricula and enhancing CSL/CFL learners' learning, their professional development should be a priority concern in research. However, studies on language teachers' development in the four leading Chinese journals are relatively few in number (48 out of 909), and one half of them are non-empirical.

As the number of Chinese language learners increases rapidly every year, more and more Chinese language teachers are needed urgently (Wang, Moloney, and Li 2013; Xu 2007). In fact, teacher shortage is one of the most significant challenges that policy makers have to face in the promotion of CIL. At least six studies have explored teacher education programmes for Chinese language teachers (e.g. Guo 2012; Li 2010; Liu 2009; Tian 2012; Yang 2006). These studies called for pre-service teacher education programmes to provide more practical, tailor-made and pedagogically informative content for course participants.

Five studies have explored language teacher cognition since what teachers think, know and believe is closely related to their classroom teaching practices (Borg 2003). 
For instance, Jiang and Hao (2010) examined and interpreted two experienced and two novice Chinese language teachers’ practical pedagogical knowledge. Teachers' beliefs, knowledge of their students and past experience were valued more by experienced teachers than by novice ones, although the novice and experienced teachers shared similar views on pedagogical knowledge. Studies have also addressed the emotional aspect of teaching. For example, Guo (2014) focused on Chinese language teachers' professional attrition. The results suggested that there was no serious indication of teacher participant burnout, but the participants did display moderate signs of emotional exhaustion, a precursor of burnout. This is something that should be taken into consideration in pre-service and ongoing in-service teacher education programmes.

\section{Conclusion}

We undertook this review to explore the contributions that Chinese scholars have made to the learning and teaching of Chinese as a second or foreign language because the Chinese language is being promoted as an effective bridge between China and the world (Xu 2007). Our review documents the notable efforts that our colleagues in the TCSL/TCFL community have made. It is particularly noteworthy that they have devoted a great deal of energy to identifying better ways of helping CSL/CFL learners acquire the language better. They have also conducted systematic inquiries to develop better language curricula, pedagogical materials and assessment tools for the promotion of Chinese as another international language. They have also argued for the need to develop appropriate pedagogical practices in response to the emerging needs and characteristics of non-Chinese learners. The need to develop teachers' professional competence and sustain their professional engagements in teaching non-Chinese learners has also been addressed in the reviewed studies - although to a limited extent. The scholarship on the learning and teaching of CSL/CFL in the four leading Chinese journals deserve the attention of those who conduct relevant research in contexts other than mainland China.

However, the studies that we reviewed in the leading Chinese journals are beset with noticeable problems. It is necessary for researchers in the field of TCSL/TCFL to conduct more rigorous research so that related research can help promote the teaching of various languages in global language education. For this reason, we would like to conclude this review with some suggestions for researchers and Chinese journals so that they can maximize the impact of their scholarship through high-quality research 
and Chinese language education in the future.

First of all, we would like to see the leading Chinese journals publishing more rigorous empirical studies so that relevant studies can inform the development and implementation of new pedagogical initiatives and practices. Researchers may draw on the similarities and differences between Chinese heritage language learners and non-Chinese heritage language learners in order to understand what the two kinds of leaners have in common and how they differ. As the teaching of Chinese is likely to take place in primary schools in many contexts, relevant studies should be conducted on teaching primary school students whose first language is not Chinese in mainland China and other parts of the world. Rigorously conducted empirical research should constitute an important part of pre-service and in-service teacher education programmes for CSL/CFL teachers.

Second, to enhance the methodological rigour of Chinese language education scholarship, we call for leading Chinese journals to serve as platforms for methodological dialogues among Chinese language teachers and researchers. These journals may include some space for teachers and researchers to undertake relevant discussions, which will help Chinese language education researchers to produce high quality research in response to Chinese language educators' practical needs. The journals will have a cross-fertilization impact on Chinese scholarship and ensure the quality of the published research studies. Fortunately, we have noted that Chinese Teaching in the World has played such role by allowing international researchers to critically monitor Chinese research scholarship.

Thirdly, we urge the leading journals and researchers to look at the development of Chinese language teachers in the context of educational reform at all levels of schooling in the world. For example, in 2015 around 12,000 Chinese language educators, including programme directors, were sent as volunteer Chinese language teachers to more than 140 countries (Hanban 2016). Unfortunately, not all the contexts that these Chinese language educators work in have been covered in research. We believe that most Chinese language educators face unique challenges in specific contexts, but they also share common challenges across contexts. Their experiences and encounters, if well documented and examined, could inform the professional development of teachers of non-Chinese learners. Therefore, we recommend that researchers working in different institutions across the world conduct collaborative studies on significant aspects of CSL/CFL teaching and learning. Leading Chinese journals may prioritize team-authored articles of this kind in their editorial processing. 
It is important for them to publish review studies that systematically evaluate particular research issues or examine empirical research on the teaching and learning of Chinese as a second or foreign language.

\section{Note}

Since 2010, Journal of College of Chinese Language and Culture of Jinan University has been renamed as Teaching Chinese to Speakers of Other Languages studies. In this review, we only used Teaching Chinese to Speakers of Other Languages studies to refer to this journal. 


\section{References}

An, F. 2015. "Analysis of fluency, grammatical complexity and accuracy of CSL writing”. Language Teaching and Linguistic Studies 3: 11-20.

An, R., and S. Zhang. 2007. "Difference in Chinese syllable acquisition among international students”. Chinese Language Learning 4: 64-71.

Bellassen, J., and M. Liao. 2013. “On epoch-making development of Chinese evaluation and teaching in France”. Teaching Chinese to Speakers of Other Languages studies 4: 4-10.

Borg, S. 2003. "Teacher cognition in language teaching: A review of research on what language teachers think, know, believe, and do”. Language teaching 36 (02): 81-109. doi: 10.1017/S0261444803001903.

Cai, L. 2009. "On the experiences of the development of Chinese education in Western Kalimantan of Indonesia”. Journal of College of Chinese Language and Culture of Jinan University 2: 8-17.

Cai, L. 2011. "A survey on the use and local compilation of Chinese teaching materials in primary schools in Indonesia”. Teaching Chinese to Speakers of Other Languages studies 3: 14-22.

Cao, X. 2008. “A comprehensive survey of the history and status quo of Chinese teaching in Northeast Asia”. Chinese Teaching in the World 3: 125-133.

Chen, J. 2006. “The status of teaching Chinese in Thailand”. Chinese Teaching in the World 3: 128-132.

Chen, L., S. She, and M. Wu. 2015. “A comparative study on Chinese learners' orthographic awareness in two different teaching models”. Language Teaching and Linguistic Studies 2: 19-25.

Chen, M. 2011. “The experimental study of the Mandarin tone category development of CSL learners with non-tone language background”. Teaching Chinese to Speakers of Other Languages studies 4: 9-15.

Chen, M. 2015. "Complexity, accuracy and fluency in Chinese as second language acquisition”. Language Teaching and Linguistic Studies 3: 1-10.

Chen, P. 2013. "Political, economical and ethnographical perspectives on teaching Chinese as a second language”. Chinese Teaching in the World 3: 400-412.

Chen, W. 2015. “An analysis of the status quo of Chinese education in Brazil”. Teaching Chinese to Speakers of Other Languages studies 2: 1-11. 
Chin, C. 2013. “On the new trend of Chinese teaching in Singapore”. Teaching Chinese to Speakers of Other Languages studies 4: 11-20.

Dai, H., and S. Yang. 2012. “The history of Chinese language teaching in Taiwan and its recent policy and measures”. Teaching Chinese to Speakers of Other Languages studies 4: 16-23.

Dai, Y. 2007. “A study on conversational repair of the Chinese second language learners”. Chinese Language Learning 6: 69-75.

Ding, A. 2007. “Japanese undergraduate students' perceptions of CSL classroom activities in a Chinese context”. Chinese Teaching in the World 1: 124-135.

Ding, A. 2014. “A study of Chinese language learners' motivation intensity”. Teaching Chinese to Speakers of Other Languages studies 3: 1-7.

Ding, X. 2006. “A study on the acquisition order of syntactic location of wh-words by foreign students”. Chinese Language Learning 5: 47-53.

Dong, J., and Z. Han. 2014. "Research on the specialized Chinese for preparatory education of overseas students in China”. Language Teaching and Linguistic Studies 4: $42-48$.

Fan, H. 2013. “The survey and analysis on foreign Chinese learners' understanding level of Chinese new words”. Teaching Chinese to Speakers of Other Languages studies 1: 29-36.

Fan, Z. 2009. “An analysis of reading anxiety of Chinese language learners from different countries”. Chinese Language Learning 3: 99-105.

Feng, L., and X. Hu. 2005. “An experimental study of the pronunciation pattern of Chinese two-syllabic words with rising tone for Korean Chinese-learning beginners”. Chinese Language Learning 4: 63-69.

Gao, X. A., Liao, Y., and Li, Y. 2014. "Empirical studies on foreign language learning and teaching in China (2008-2011): A review of selected research”. Language Teaching 47 (01): 56-79. doi: 10.1017/S0261444813000414.

Gao, Y., Li, L., and Lü, J. 2001. “Trends in research methods in applied linguistics: China and the West”. English for Specific purpose 20: 1-14.

Geng, Y. 2005. "Sinology and Chinese language education in Frankfurt University”. Chinese Teaching in the World 4: 98-101.

Gong, Z., W. Ding, and L. Chen. 2008. "The status of Chinese education in the universities in Bangladesh”. Chinese Teaching in the World 4: 133-140.

Guo, C. 2005. “On the problems of Chinese education in Japanese universities”. Chinese Teaching in the World 4: 91-97. 
Guo, F. 2012. “Considerations on the training of overseas Chinese teaching staff”. Language Teaching and Linguistic Studies 2: 33-38.

Guo, R. 2014. “Job burnout of Chinese teachers”. Language Teaching and Linguistic Studies 6: 20-28.

Hanban. 2016. 2015 Confucius Institute Annual Development Report. Beijing: Confucius Institute Headquarters.

Hao, L. 2013. “A study of authenticity, typicality and appropriateness in TCFL textbooks”. Teaching Chinese to Speakers of Other Languages studies 3: 41-49.

Hao, Y., and Z. Wang. 2013. "A study on the requirements of learners in L1 environment for Chinese language learners' dictionary”. Teaching Chinese to Speakers of Other Languages studies 3: 50-57.

Hu, G. 2005. "English language education in China: Policies, progress, and problems”. Language policy 4 (01): 5-24. doi: 10.1007/s10993-004-6561-7.

Huang, B., and X. Liao. 2002. Modern Chinese (the $3^{\text {rd }}$ Edition). Beijing: Higher Education Press.

Huang, H. 2005. “The current situation of Thailand universities' Chinese language teaching and instructors". Journal of College of Chinese Language and Culture of Jinan University 3: 1-9.

Huang, Y., S. Yang, L. Gao, and X. Cui. 2005. "The acquisition of Chinese reflexive "Ziji" by English and Japanese speakers”. Chinese Language Learning 5: 49-60.

Huang, Y., S. Yang, L. Gao, W. Zhang, and X. Cui. 2007. "The L2 acquisition of the Chinese Bei-construction”. Chinese Teaching in the World 2: 76-90.

Huang, Z., and X. Xiao. 2012. “Acquisition of Ba-construction of Korean students based on interlanguage corpus”. Chinese Language Learning 1: 71-79.

Jiang, X. 2007. “An experimental study on the effect of the method of teaching the learner to recognize characters more than writing”. Chinese Teaching in the World 2: 91-97.

Jiang, X., \& L. Hao. 2010. "A case study of the practical knowledge of TCFL teachers”. Chinese Teaching in the World 3: 394-405.

Lei, L. 2006. “Chinese language education in Korea’s Samsung Company”. Chinese Teaching in the World 1: 122-126.

Li, B. 2005. "An analysis of teaching Chinese for Chinese heritage students in Canada”. Chinese Teaching in the World 1: 106-111.

Li, D., and J. Zhang. 2006. "Investigation on the Validity of Chinese Proficiency Test for Minorities in China”. Chinese Language Learning 3: 74-80. 
Li, H. 2010. “A study on the development and problems of Chinese teaching in Thai universities under the Sino-Thai cooperation”. Teaching Chinese to Speakers of Other Languages studies 1: 3-9.

Li, Q. 2010. “A study of the training principles and implementation focus of MTCSOL”. Teaching Chinese to Speakers of Other Languages studies 3: 1-7.

Li, Q. 2015. “Linguistic standards of Chinese as a foreign language”. Language Teaching and Linguistic Studies 5: 1-11.

Li, Q., and X. Gong. 2015. "The general, regional, first-language-type and country-type of textbooks of Chinese as a foreign language”. Chinese Language Learning 1: 76-84.

Li, X, and Li, J. 2014. "Validity analysis of Spoken Chinese Test”. Chinese Teaching in the World 1: 103-112.

Li, Y., and X. Deng. 2005. "Study of selecting and sequencing of Ba sentence patterns”. Language Teaching and Linguistic Studies 3: 50-58.

Li, Y., and X. Xu. 2009. “A Case Study of Misuse of bu (不) and mei (没) by Englishspeaking Learners of Chinese”. Journal of College of Chinese Language and Culture of Jinan University 3: 20-26.

Lin, Q. 2011. “Research on Korean students' acquisition of structures of Zai+NL”. Chinese Language Learning 3: 89-98.

Liu, F. 2012. “The analysis on Japanese students' Chinese language learning strategies”. Chinese Language Learning 4: 97-103.

Liu, L. 2006. "Discourse markers' acquisition and misusing in intercultural communication”. Chinese Language Learning 4: 50-57.

Liu, S. 2009. “On a new teaching model for CSL listening”. Journal of College of Chinese Language and Culture of Jinan University 2: 18-25

Liu, Y. 2008. "A comparative study on the characteristics of the Japanese and Chinese as a second language”. Journal of College of Chinese Language and Culture of Jinan University 4: 31-41.

Liu, Y. 2009. “A comparative study on teacher education of teaching Chinese and Japanese as a second language”. Chinese Language Learning 3: 85-92.

Liu, Y. 2014. “On Chinese Flagship Program in the United States: The model of Brigham Young University Chinese Flagship Program”. Teaching Chinese to Speakers of Other Languages studies 2: 37-42.

Lochmiller, C. R., and Lester, J. N. 2016. An Introduction to Educational Research:

Connecting Methods to Practice. SAGE Publications. 
Luan, Y. 2013. "An empirical study on the causes of misuse of "S+gei+NP1+V+NP2" as double objects sentence". Teaching Chinese to Speakers of Other Languages studies 3: 20-27.

Mao, Y. 2010. "A study of short term Chinese teaching model for learners from overseas enterprises”. Chinese Teaching in the World 1: 103-111.

Mei, L. 2011. "Perceptual assimilation and discrimination of Chinese affricates by Thai learners”. Chinese Teaching in the World 2: 276-288.

Mo, F. 2007. "Enhancing the exercises of communication to reinforce the consciousness of Vietnamese students using Ba-sentence”. Journal of College of Chinese Language and Culture of Jinan University 3: 31-42.

Moloney, R., and Xu, H. 2015. "Transitioning beliefs in teachers of Chinese as a foreign language: An Australian case study”. Cogent Education, 2: 1-15, doi: 10.1080/2331186X.2015.1024960.

$\mathrm{Ni}$, C. 2007. “An analysis of the learning needs of international learners of Chinese in China”. Language Teaching and Linguistic Studies 1: 68-76.

Peng, B. 2012. “On functions of Chinese newspaper in Chinese education: The case of Chinese newspaper in Malaysia”. Teaching Chinese to Speakers of Other Languages studies 4: 10-15.

Peng, S. 2008. "A study on the acquisition of the Bei sentence including a verb followed by an object”. Chinese Language Learning 2: 91-99.

Perfetti, C. A., and Dunlap, S. 2008. "Learning to read: General principles and writing system variations”. Learning to read across languages, 13-38. New York: Routledge. Qian, Q. 2011. “Chinese language classes in American public schools”. Chinese Teaching in the World 1: 110-119.

Qian, Y. 2010. “A comparative study of Chinese reading strategies among Chinese and Korean students”. Teaching Chinese to Speakers of Other Languages studies 3: 42-29.

Shao, J. 2013. “Teaching Chinese personal anaphora to second language learners”. Chinese Teaching in the World 4: 537-547.

Shen, H. H., and Jiang, X. 2013. "Character reading fluency, word segmentation accuracy, and reading comprehension in L2 Chinese”. Reading in a Foreign Language 25(1): 1-25.

Shen, H. H., and Xu, W. 2015. “Active learning: qualitative inquiries into vocabulary instruction in Chinese L2 classrooms”. Foreign Language Annals 48(1): 82-99. doi: 
10.1111/flan.12137.

Shen, S. 2006. “The orientation of the intensive business Chinese course”. Language Teaching and Linguistic Studies 5: 75-80.

Shen, S. 2014. "Research on practice teaching of business Chinese to foreign students”. Language Teaching and Linguistic Studies 1: 45-50.

Su, D. 2010. “On the construction-chunk approach”. Chinese Language Learning 2: 83-90.

Su, D., and J. Lu. 2010. “Construction-chunk approach for syntactic analysis and second language pedagogy”. Chinese Teaching in the World 4: 557-567.

Tao, J. 2012. "The analysis on the Chinese curriculum of IB diploma programme and the teaching material compilation strategies”. Teaching Chinese to Speakers of Other Languages studies 2: 1-8.

Tian, Y. 2012. "Implications of MTESOL curricular in UK for programme design of MTCSOL”. Chinese Teaching in the World 6: 276-288.

Wang, D., Moloney, R., and Li, Z. (2013). “Towards internationalising the curriculum: A case study of Chinese language teacher education programs in China and Australia”. Australian Journal of Teacher Education, 38(9): 116-135. doi: 10.14221/ajte.2013v38n9.8.

Wang, J. 2005. “On the design of CLCSP based on need analysis”. Language Teaching and Linguistic Studies 5: 55-59.

Wang, J. 2005. "On the design of CLCSP based on the need analysis”. Language Teaching and Linguistic Studies 5, 55-59.

Wang, J. 2008. “The language standards of the international promotion of Chinese language and the competitive strategies”. Language Teaching and Linguistic Studies 1: 65-72.

Wang, M. 2005. “A study on foreign learners' acquisition of Bi structure”. Journal of College of Chinese Language and Culture of Jinan University 3: 28-35

Wang, Q., and Z. Chu. 2009. “The features of Chinese classes at Indiana University Bloomington". Journal of College of Chinese Language and Culture of Jinan University 3: 1-4.

Wang, W., and Gao, X. 2008. "English language education in China: A review of selected research”. Journal of Multilingual and Multicultural Development 29(05): 380-399. doi: 10.1080/01434630802147908.

Wang, X. 2006. “A study on the validity of HSK”. Language Teaching and Linguistic Studies 6: 49-56. 
Wang, Y. 2008. "The status quo, problems and their solutions of Chinese teaching in primary schools and high schools of Thailand”. Journal of College of Chinese Language and Culture of Jinan University 4: 9-16.

Wen, X. 2011. "Challenges and changes in teaching Chinese as a second language in the USA”. Chinese Teaching in the World 4: 538-552.

Wu, M., and L. Chen. 2012. "A longitudinal study of intentional and incidental vocabulary learning”. Teaching Chinese to Speakers of Other Languages studies 3: 11-18.

Wu, M., D. Gao, X. Xiao, and R. Zhang. 2006. “A study of learning reading and writing Chinese characters by CSL learners from Korea, Japan and Western countries”. Language Teaching and Linguistic Studies 6: 64-71.

Wu, Y. 2008. "A case study on oral Chinese learning strategies used by Italian learners in different social environments”. Chinese Teaching in the World 4: 88-100. Wu, Y., and J. Yang. 2008. "On the mode of rapid promoting Chinese teaching in Thailand”. Chinese Teaching in the World 4: 125-132.

Wu, Y., and Y. Chen. 2006. "Comparative research on learning strategies for listening between the effective and ineffective Learners”. Chinese Language Learning 2: 58-64.

Xiao, P., and Z. Liu. 2013. “An analysis of Indonesian students' specific Chinese confusable words and their L1 influencing factors". Teaching Chinese to Speakers of Other Languages studies 1: 37-45.

Xie, F. 2010. “A corpus-based study on the acquisition of Shi......de sentence”. Language Teaching and Linguistic Studies 2: 17-24.

$\mathrm{Xu}, \mathrm{C}$. 2009. "The developmental process of foreign students discriminating form errors of Chinese characters”. Chinese Language Learning 6: 71-78.

$\mathrm{Xu}, \mathrm{L}$. 2007. "The situation and mission of Chinese international promotion”. Chinese Teaching in the World 2: 106-110.

Xu, X., and L. Yao. 2014. “A survey of students' motivation with language-character integration approach and language-character separation approach”. Teaching Chinese to Speakers of Other Languages studies 3: 8-16.

Yang, H. 2005. “Chinese language education in Inje University”. Chinese Teaching in the World 4: 102-104.

Yang, H. 2010. “Tapping the students’ potential by encouraging their active thinking”. Chinese Teaching in the World 4: 568-576.

Yang, L. 2006. “The study of Chinese language teacher training in Laos”. Chinese 
Teaching in the World 4: 116-120.

Yao, D. 2014. "University of Hawaii and Chinese language instruction in the United States”. Teaching Chinese to Speakers of Other Languages studies 1: 9-14.

Yu, Z. 2012. "Debate on Chinese as one of the most difficult languages in the world". Language Teaching and Linguistic Studies 4: 38-45.

Yuan, L. 2009. “An error analysis of cohesive devices in Chinese discourses by English-speaking learners of Chinese”. Journal of College of Chinese Language and Culture of Jinan University 4: 27-34.

Zhang, J. 1992. Modern Chinese character tutorial. Beijing: Modern Publishing House.

Zhang, J. 2008. "On the stroke effect, word frequency effect and morpheme frequency effect in the recognition of Chinese one-character word by foreign students”. Journal of College of Chinese Language and Culture of Jinan University 1: 22-29.

Zhang, L. 2006. “A need analysis in teaching business Chinese”. Language Teaching and Linguistic Studies 3: 55-60.

Zhang, L. 2015. “An investigation into American college students' motivation for Chinese learning”. Teaching Chinese to Speakers of Other Languages studies 3: 6-10. Zhang, L., S. Yang, and H. Zhang. 2008. "Using slides to teaching senior Chinese text readings". Chinese Teaching in the World 4: 114-124.

Zhang, X. 2008. "The investigation and analysis of spoken Chinese teaching in Colombia”. Journal of College of Chinese Language and Culture of Jinan University 4: $17-25$.

Zhao, L. 2008. “Considerations on establishing task-based teaching system of oral Chinese”. Language Teaching and Linguistic Studies 3: 64-69.

Zhao, W., L. Zhang, and L. Yao. 2013. “The basic Characteristics and methods of assessment in K-12 Chinese teaching in the USA". Chinese Teaching in the World 1: 117-127.

Zhao, X. 2015. Support attracts top brains to China. China Daily, February 27. http://english.gov.cn/policies/policy_watch/2015/02/27/content_281475062450341.ht m.

Zheng, L. 2015. “Acquisition of Chinese object-taking intransitive constructions by English-speaking learners”. Chinese Teaching in the World 3: 393-404.

Zhou, J., and H. Xie. 2007. “On Chinese word boundary parsing ability of foreign students”. Chinese Language Learning 2: 70-76. 
Zhou, X., and N. Chen. 2013. “A study on the localization of single-edition-multi-version Chinese textbooks vis-à-vis overseas Chinese textbooks”. Chinese Teaching in the World 2: 268-277.

Zhou, X., Y. Luo, and L. Zhang. 2010. “A systematic and comparative investigation into Chinese culture textbooks”. Language Teaching and Linguistic Studies 5: 1-7. Zhu, Q. 2007. “An error analysis of blends made by international learners of Chinese”. Chinese Language Learning 3: 70-76.

Zong, S., X. Zhu, and W. Liu. 2012. “On length approach and its application in TCSL writing” Chinese Teaching in the World 2: 254-265.

$\mathrm{Zu}, \mathrm{X}$. 2008. "Corrective feedback in Chinese language classrooms". Chinese Language Learning 1: 93-100.

“汉语国际推广简史：从对外汉语教学到孔子学院”. 中国网, September 3, 2009. http://www.china.com.cn/international/txt/2009-09/03/content_18456777_2.htm. 


\section{Appendix 1: Detailed numbers of studies with different methodological trends and about major issues}

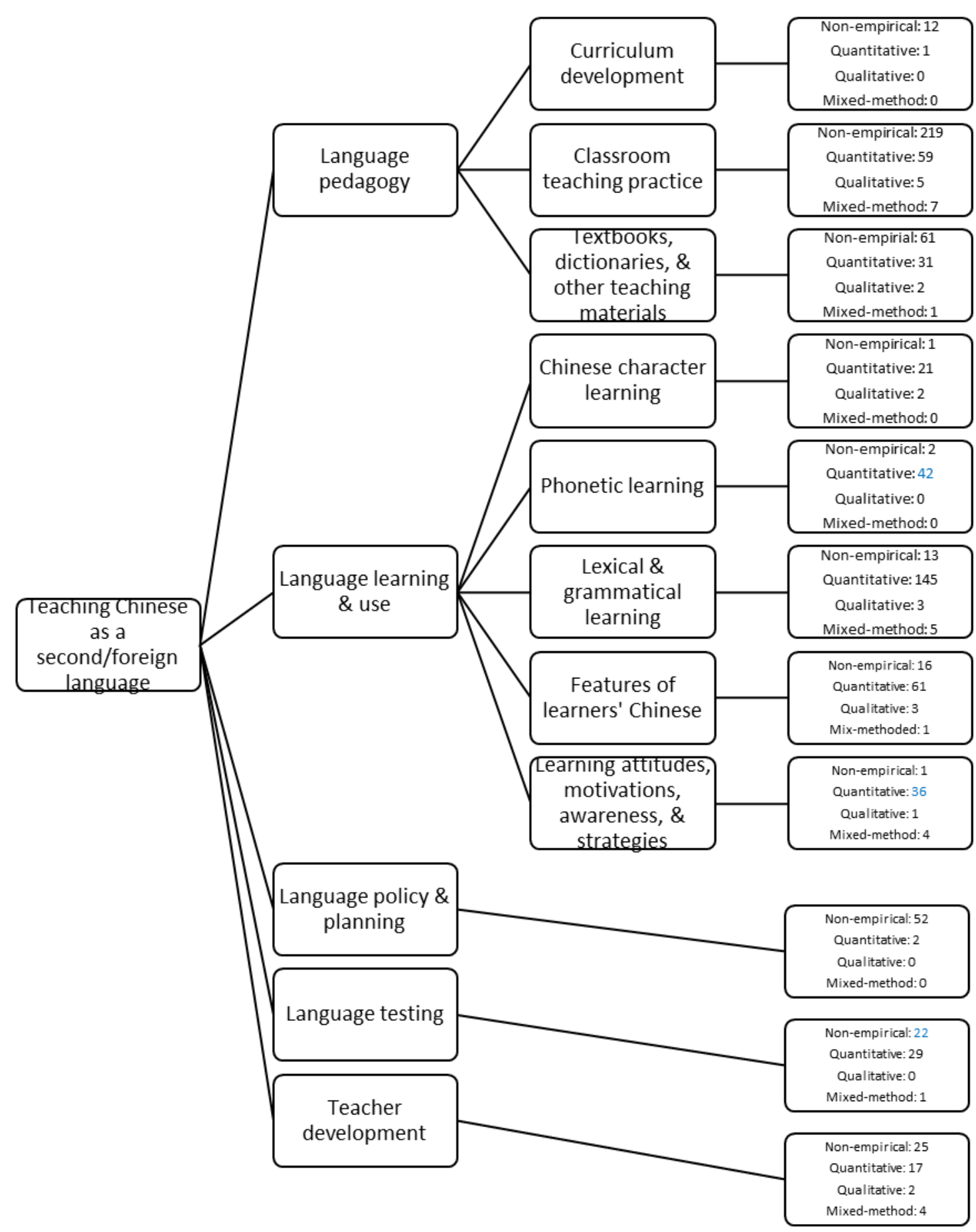


Table 1 Journals reviewed

\begin{tabular}{|c|c|c|c|c|}
\hline No. & Chinese name & English name & $\begin{array}{l}\text { Journal base } \\
\text { (affiliated } \\
\text { institution, if } \\
\text { any) }\end{array}$ & $\begin{array}{l}\text { Compound \& } \\
\text { Comprehensive } \\
\text { impact factor }\end{array}$ \\
\hline 1 & 汉语学习 & $\begin{array}{l}\text { Chinese Language } \\
\text { Learning (CLL) }\end{array}$ & $\begin{array}{l}\text { Yanbian } \\
\text { University }\end{array}$ & $\begin{array}{l}1.062 \& \\
0.410\end{array}$ \\
\hline 2 & $\begin{array}{l}\text { 语言教学与 } \\
\text { 研究 }\end{array}$ & $\begin{array}{l}\text { Language Teaching and } \\
\text { Linguistic Studies } \\
\text { (LT\&LS) }\end{array}$ & $\begin{array}{l}\text { Beijing Language } \\
\text { and Culture } \\
\text { University }\end{array}$ & $\begin{array}{l}1.350 \& \\
0.583\end{array}$ \\
\hline 3 & $\begin{array}{l}\text { 华文教学与 } \\
\text { 研究 }\end{array}$ & $\begin{array}{l}\text { Teaching Chinese to } \\
\text { Speakers of Other } \\
\text { Languages (TCSOL) } \\
\text { Studies (TCSOL) }\end{array}$ & Ji’nan University & $\begin{array}{l}0.806 \& \\
0.306\end{array}$ \\
\hline 4 & $\begin{array}{l}\text { 世界汉语教 } \\
\text { 学 }\end{array}$ & $\begin{array}{l}\text { Chinese Teaching in the } \\
\text { World (CTW) }\end{array}$ & $\begin{array}{l}\text { Beijing Language } \\
\text { and Culture } \\
\text { University }\end{array}$ & $\begin{array}{l}1.924 \& \\
0.804\end{array}$ \\
\hline
\end{tabular}

Table 2 Methodological trends (2005-2015) (N=909)

\begin{tabular}{lllll}
\hline \hline & $\begin{array}{l}\text { Non-empirical } \\
\text { studies }\end{array}$ & $\begin{array}{l}\text { Quantitative } \\
\text { studies }\end{array}$ & $\begin{array}{l}\text { Qualitative } \\
\text { studies }\end{array}$ & $\begin{array}{l}\text { Mix-method } \\
\text { studies }\end{array}$ \\
\hline 2005 & $38(52.1 \%)$ & $32(43.8 \%)$ & $3(4.1 \%)$ & 0 \\
2006 & $47(57.3 \%)$ & $30(36.7 \%)$ & $3(3.7 \%)$ & $2(2.4 \%)$ \\
2007 & $35(47.3 \%)$ & $34(45.9 \%)$ & $2(2.7 \%)$ & $3(4.1 \%)$ \\
2008 & $53(60.2 \%)$ & $31(35.2 \%)$ & $1(1.1 \%)$ & $3(3.4 \%)$ \\
2009 & $50(57.5 \%)$ & $34(39.1 \%)$ & 0 & $3(3.4 \%)$ \\
2010 & $47(55.3 \%)$ & $34(40.0 \%)$ & $3(3.5 \%)$ & $1(1.2 \%)$ \\
2011 & $42(51.9 \%)$ & $37(45.7 \%)$ & 0 & $2(2.5 \%)$ \\
2012 & $33(36.3 \%)$ & $55(60.4 \%)$ & 0 & $3(3.3 \%)$ \\
2013 & $29(34.9 \%)$ & $52(62.7 \%)$ & $1(1.2 \%)$ & $1(1.2 \%)$ \\
2014 & $33(36.7 \%)$ & $51(56.7 \%)$ & $3(3.3 \%)$ & $3(3.3 \%)$ \\
\hline \hline
\end{tabular}




\begin{tabular}{lllll}
\hline \hline 2015 & $18(22.8 \%)$ & $57(72.2 \%)$ & $2(2.5 \%)$ & $2(2.5 \%)$ \\
\hline \hline
\end{tabular}

Table 3 Methodological trends in four journals ( $\mathrm{N}=909)$

\begin{tabular}{llllll}
\hline \hline & CLL & LT\&LS & TCSOL & CTW & $\begin{array}{l}\text { Total } \\
(909)\end{array}$ \\
& $(182)$ & $(261)$ & $(265)$ & $(201)$ & 425 \\
Non-empirical & 86 & 112 & 122 & 105 & $(46.5 \%)$ \\
studies & $(46.7 \%)$ & $(42.6 \%)$ & $(46.0 \%)$ & $(52.2 \%)$ & 447 \\
Quantitative & 91 & 141 & 130 & 85 & $(49.0 \%)$ \\
studies & $(49.5 \%)$ & $(53.6 \%)$ & $(49.1 \%)$ & $(42.3 \%)$ & 18 \\
Qualitative & 3 & 5 & 7 & 3 & $(2.0 \%)$ \\
studies & $(1.6 \%)$ & $(1.9 \%)$ & $(2.6 \%)$ & $(1.5 \%)$ & 23 \\
Mixed-method & 4 & 5 & 6 & 8 & $(2.5 \%)$ \\
studies & $(2.2 \%)$ & $(1.9 \%)$ & $(2.3 \%)$ & $(4.0 \%)$ & \\
\hline \hline
\end{tabular}

